\title{
Essais
}

ESSAIS

Revue interdisciplinaire d'Humanités

Hors-série 6 | 2021

Agrobiodiversité et territoires

\section{La conservation des variétés anciennes de fruitiers en régions Nouvelle Aquitaine et Occitanie}

Rencontre avec Hubert Didier

Conservation of old fruit varieties in the regions of New Aquitaine and Occitania

Meeting with Hubert Didier

\section{OpenEdition}

Journals

Édition électronique

URL : https://journals.openedition.org/essais/7949

DOI : 10.4000/essais.7949

ISSN : 2276-0970

Éditeur

École doctorale Montaigne Humanités

Édition imprimée

Date de publication : 1 mars 2021

Pagination : $173-176$

ISBN : 978-2-492780-00-4

ISSN : $2417-4211$

Référence électronique

"La conservation des variétés anciennes de fruitiers en régions Nouvelle Aquitaine et Occitanie », Essais [En ligne], Hors-série 6 | 2021, mis en ligne le 16 mars 2021, consulté le 18 janvier 2023. URL http://journals.openedition.org/essais/7949; DOI : https://doi.org/10.4000/essais.7949 


\title{
La conservation des variétés anciennes de fruitiers en régions Nouvelle Aquitaine et Occitanie
}

\author{
Entretien avec Hubert Didier ${ }^{1}$
}

Hubert Didier est salarié du Conservatoire Végétal Régional d'Aquitaine (CVRA) de Montesquieu, responsable notamment de l'entretien et du suivi des collections ainsi que des 35 vergers en convention "Sites d'accueil » du Conservatoire.

\section{Pourriez-vous faire un rapide historique ainsi qu'une présentation du CVRA?}

Le CVRA est une association loi 1901, créée en 1996 par le Conseil Régional d'Aquitaine pour officialiser le travail réalisé au sein du Parc Naturel Régional des Landes de Gascogne et du CIREA par Evelyne Leterme, actuelle directrice, à partir de 1979. Sa mission était de sauvegarder le patrimoine fruitier local existant ou en voie de disparition à cause notamment de la spécialisation agricole et de l'arrêt de l'arboriculture traditionnelle. Grâce à un partenariat entre le Muséum National d'Histoire Naturelle, le Parc Naturel Régional des Landes de Gascogne, la Région Nouvelle Aquitaine anciennement Région Aquitaine et l'Université de Bordeaux, un centre de Ressources Génétiques est constitué. Les actions de ce centre s'articulent autour de 4 axes, selon la définition proposée par Jean Pernes, généticien au CNRS : prospection, conservation (mise en collection), évaluation et diffusion. Deux grandes phases suivent cette création : de 1979 à 1995 s'opèrent les principales collectes et constitutions des collections à l'écomusée de Sabres, sur un verger de deux hectares et demi ; puis, entre 1995 et 1996, les collections sont déplacées pour être plantées à Montesquieu (47) sur le site actuel.

1 Dans le cadre de sa participation au séminaire des 14 et 15 mai 2019. 


\section{Quel est le fonctionnement de cette structure ? Comment sont impli- qués les partenaires du territoire Aquitain?}

Le CVRA fonctionne sur 3 pôles complémentaires, le CVRA qui assure la conservation des ressources génétiques, l'Association de soutien et les sites d'accueils.

L'association du CVRA siège au Domaine de Barolle, avec 6 à 8 salariés employés selon les périodes ; cette équipe est renforcée par plus de 150 bénévoles actifs, se chargeant notamment des activités de diffusion et d'exposition (pour un équivalent de 6 emplois à l'année), pour la plupart consommateurs ou partenaires membres de l'Association de Soutien au CVRA. Le fond génétique du CVRA est constitué de plus de 2000 accessions (2 000 individus de variétés et populations variétales différentes) réparties entre le verger du domaine et les 35 sites d'accueil actifs, associés via des conventions de 5 ans et présents sur 7 départements. Les missions et actions sont discutées et votées par le Conseil d'Administration constitué de 30 membres et 5 collèges : les collectivités territoriales (Départements, Région), les représentants de l'Association de Soutien au Conservatoire, les représentants des sites d'accueil (Mairies, vergers d'entreprises, vergers de particuliers à vocation pédagogique ou paysagère), et les autres organismes régionaux (associations partenaires). Les orientations scientifiques sont instaurées quant à elles par un Conseil Scientifique à réunion annuelle, d'environ 15 personnes venant de toute la France, scientifiques travaillant principalement sur l'agroécologie, la conservation du patrimoine domestique et l'étude de la qualité alimentaire.

Le financement provient à $80 \%$ de fonds propres principalement issus des productions de la pépinière : ventes de fruitiers avec un catalogue de 325 variétés issues de 25 espèces, ventes de fruits frais de petites séries ( 4 à 5 arbres par variété) et de produits transformés par les bénévoles (gelées et confitures). Le CVRA propose également des stages grand public ou professionnels autour de la taille, de la greffe, de la préparation des sols et plus largement aux pratiques agroécologiques, et génère un autofinancement complémentaire lié à la vente d'ouvrages et publications sur les thématiques associées (site internet et Fête de la Nature). De plus les conventions avec les sites d'accueil et prestation permettent de financer un poste.

\section{Quelles sont concrètement les actions du CVRA pour la sauvegarde du patrimoine végétal fruitier?}

Les missions sont orientées vers la collecte et la caractérisation des variétés mises en collection. Ces collections sont implantées sous forme d'arbres sur le site de Montesquieu, dans un verger à haute densité de 17 hectares avec 2000 accessions de plus de 7500 arbres palissés (4 ou 5 individus par variété) ainsi que sur les vergers conventionnés répartis sur tout le territoire aquitain. 
Cette caractérisation des variétés capitalise plusieurs informations, s'axant sur plusieurs critères comme la phénologie, avec un classement selon la date de maturité des fruits générant un carnet de récolte renseignant également les dates de débourrement ou de foliation. Tous les cycles et moments cruciaux de l'arbre sont ainsi observés et renseignés pour servir ensuite les pratiques culturales. On observe également les comportements des arbres exposés aux parasites et maladies, leurs réactions (adaptations ou blocages) aux changements climatiques. De nouveaux outils comme l'analyse moléculaire, financée à échelle nationale ou par fonds propres, permettent aujourd'hui de voir les mouvements des variétés à échelle française, européenne ou mondiale et ainsi les ressemblances génétiques entre variétés. L’analyse biochimique, comme les taux de vitamine $\mathrm{C}$, permettrait de proposer des variétés intéressantes pour certaines filières agroalimentaires. Enfin, l'analyse des conduites variétales permet de poser les méthodes de conduite qui optimisent la production et le calibre des fruits.

\section{En quoi les actions du CVRA contribuent-elles à une gestion paysa- gère et environnementale durable des territoires aquitains ?}

Le CVRA mène ses actions grâce à un ensemble de pratiques agroécologiques qui se veulent adaptées aux " terroirs " locaux. Par exemple, à échelle du verger, la logique écologique vise la diversité des milieux, la diversité intraspécifique (plusieurs individus de la même espèce) et interspécifique (plusieurs espèces et plusieurs variétés). Les vergers sont ainsi issus des collectes de patrimoines à la fois génétiques, culturels et historiques, les modes de culture et les connaissances autour des variétés étant également répertoriés et reproduits. Le CVRA, au-delà de promouvoir certaines pratiques issues de savoir-faire traditionnels, expérimente les conditions de reproduction de ces modèles de culture. Par exemple, la forme paysagère typique du Sud-Ouest mêlant les joualles ou canses, vignes palissées plantées d'arbres fruitiers où s'entremêlent des cultures vivrières de tabac, pommes de terre ou maïs ; les vignes sur hautains en Haute-Garonne, parcelles de vignes cultivées sur des érables champêtres taillés à 2 mètres de hauteur, à entre-rangs destinés aux cultures vivrières; les systèmes d'arboriculture mêlée à la tulipe agenaise, espèce symbiotique à caractère patrimonial anciennement cultivée pour son caractère ornemental ; ou encore les haies fruitières paysagères ou de production, en systèmes bocagers, aux linéaires constituant des corridors écologiques qui sont renforcés par le Conservatoire de plantes compagnes qui rentabilisent le système global. Tous ces modèles sont expérimentés sur les vergers sites d'accueil afin de les adapter à l'économie actuelle et d'en tester la reproductibilité.

Le verger du domaine de Barolle est en conversion biologique depuis 2 ans, sollicitant notamment la pulvérisation d'éliciteurs stimulant les défenses immunitaires des arbres. À terme, le CVRA souhaite asseoir son importance avec la création d'un label et l'obtention de l'agrément national de gestion- 
naire de ressources phytogénétiques (janvier 2020), tout en accentuant le renouvellement des collections fruitières, le développement des sites d'accueil et les prestations de conseils, avec une sélection adaptée aux besoins agricoles et aux ressources génétiques locales.

Les implantations paysagères d'arbres fruitiers sont devenues la marque du Conservatoire végétal d'Aquitaine au sein de ses sites d'accueils, de plus en plus souvent au cœur des villes et des villages, objet d'une formation spécifique auprès de l'École d'Architecture et du Paysage de Bordeaux et d'une implantation au Potager du Roi à Versailles. 